\title{
UNRAMIFIED COVERS OF GALOIS COVERS OF LOW GENUS CURVES
}

\author{
BJORN POONEN
}

\begin{abstract}
Let $X \rightarrow Y$ be a Galois covering of curves, where the genus of $X$ is $\geq 2$ and the genus of $Y$ is $\leq 2$. We prove that under certain hypotheses, $X$ has an unramified cover that dominates a hyperelliptic curve; our results apply, for instance, to all tamely superelliptic curves. Combining this with a theorem of Bogomolov and Tschinkel shows that $X$ has an unramified cover that dominates $y^{2}=x^{6}-1$, if char $k$ is not 2 or 3 .
\end{abstract}

\section{Introduction}

1.1. Definitions. Let $k$ be an algebraically closed field. Let $p$ be the characteristic of $k$ (we allow the case $p=0$ ). In this paper, a curve is a smooth, projective, integral, 1-dimensional variety over $k$. If we write an affine equation for a curve, its smooth projective model is implied. We write $g(X)$ for the genus of a curve $X$. By an unramified cover of a curve $X$, we mean a curve $Z$ with a finite étale morphism $Z \rightarrow X$. As usual, one says that $X$ dominates $Y$ if there is a rational map $X \rightarrow Y$ whose image is Zariski dense in $Y$; for curves (satisfying our hypotheses), this is equivalent to the existence of a surjective morphism.

Definition 1.1. Let $X$ and $Y$ be curves. Following [Bogomolov-Tschinkel2004couniformization], we write $X \Rightarrow Y$ if there exists an unramified cover $Z$ of $X$ such that $Z$ dominates $Y$. Write $X \Leftrightarrow Y$ if $X \Rightarrow Y$ and $Y \Rightarrow X$.

The relation $\Rightarrow$ is reflexive and transitive. For any $X$, we have $X \Rightarrow \mathbb{P}^{1}$. On the other hand, $\mathbb{P}^{1}$ has no nontrivial unramified covers; thus $\mathbb{P}^{1} \Rightarrow X$ only if $X \simeq \mathbb{P}^{1}$. Hence the relation $\Rightarrow$ is not symmetric.

Remark 1.2. One motivation for introducing the relation $\Rightarrow$ arises from arithmetic geometry. Suppose $X, Y$ are curves over a number field $F$. If $X \Rightarrow Y$, then by [Chevalley-Weil1930], the problem of determining the $F$-points on $X$ can be reduced to finding the $F^{\prime}$-points on $Y$ for some effectively computable finite extension $F^{\prime}$ of $F$.

Received by the editors December 30, 2004.

2000 Mathematics Subject Classification. Primary 14H30; Secondary 14H45.

Key words and phrases. Galois cover, unramified cover, Abhyankar's lemma, superelliptic curve.

This research was supported by NSF grant DMS-0301280 and a Packard Fellowship. 
1.2. Previous results. Belyı $[$ Belyi1979] proved that every curve over $\overline{\mathbb{Q}}$ admits a morphism to $\mathbb{P}^{1}$ ramified only above $\{0,1, \infty\}$. Almost immediately thereafter, Manin proved that Belyıı's Theorem implies the following theorem:

Theorem 1.3 ([Bogomolov-Husemoller2000, Proposition 7.1]). For any curve $X$ over $\overline{\mathbb{Q}}$, there exists $N \geq 1$ such that the modular curve $X(N)$ satisfies $X(N) \Rightarrow$ $X$.

Call a curve $X$ hyperelliptic if there exists a degree-2 map $X \rightarrow \mathbb{P}^{1}$ and $g(X) \geq 2$.

Theorem 1.4 ([Bogomolov-Tschinkel2002-unramified, Theorem 1.7]). If $X$ is a hyperelliptic curve over $\overline{\mathbb{F}}_{p}$, and $Y$ is any curve over $\overline{\mathbb{F}}_{p}$, then $X \Rightarrow Y$.

Let $C_{n}$ be (the smooth projective model of) the curve $y^{2}=x^{n}-1$.

Theorem 1.5 ([Bogomolov-Tschinkel2002-unramified, Proposition 1.8]). Suppose $p \neq 2,3$. If $X$ is a hyperelliptic curve over $k$, then $X \Rightarrow C_{6}$.

Theorem 1.6 ([Bogomolov-Tschinkel2004-couniformization]). Suppose $k=\overline{\mathbb{Q}}$. For any $m \geq 5$ and $n \in\{2,3,5\}$, we have $C_{m} \Leftrightarrow C_{m n}$.

Proof. The direction $C_{m n} \Rightarrow C_{m}$ is trivial. For $C_{m} \Rightarrow C_{m n}$, the case $m \geq 6$ is [Bogomolov-Tschinkel2004-couniformization, Theorem 1.2], and the case $m=5$ is a consequence of [Bogomolov-Tschinkel2004-couniformization, Corollary 2.8].

1.3. New results. If $X \rightarrow Y$ is a dominant morphism of curves, call $X$ a Galois cover of $Y$ if the corresponding function field extension $k(X)$ over $k(Y)$ is Galois (thus we do not require that $X$ be unramified over $Y$ ). If moreover $\operatorname{Gal}(k(X) / k(Y))$ is cyclic, then call $X$ a cyclic cover of $Y$. If $G$ is a subgroup of Aut $X$, then $X / G$ denotes the curve whose function field is the fixed field $k(X)^{G}$.

Theorem 1.7. Let $X$ be a curve. Let $G$ be a subgroup of $\operatorname{Aut}(X)$ of order not divisible by the characteristic of $k$. Let $Y=X / G$. Suppose $g(X) \geq 2 \geq g(Y)$. Suppose in addition that at least one of the following holds:

(1) $g(Y) \in\{1,2\}$.

(2) $G$ is solvable.

(3) There are two distinct points of $Y$ above which the ramification indices have a nontrivial common factor.

(4) There are three points of $Y$ above which the ramification indices are divisible by $2,3, \ell$, respectively, where $\ell$ is a prime with either $\ell \leq 89$ or

$$
\ell \in\{101,103,107,131,167,191\} .
$$

Then $X \Rightarrow H$ for some hyperelliptic curve $H$.

Corollary 1.8. If in addition to the hypotheses of Theorem 1.7 we have $p \neq 2,3$, then $X \Rightarrow C_{6}$.

Proof. Combine Theorem 1.7 with Theorem 1.5, and use transitivity of $\Rightarrow$. 
Call a curve $X$ tamely superelliptic if $X$ is a cyclic cover of $\mathbb{P}^{1}$ of degree not divisible by $p$, and $g(X) \geq 2$. These are the curves of genus $\geq 2$ with equations of the form $y^{n}=f(x)$ with $p \nmid n$.

Corollary 1.9. If $X$ is tamely superelliptic, then $X \Rightarrow H$ for some hyperelliptic curve $H$.

Proof. Theorem 1.7 applies because the Galois group is solvable.

\section{Lemmas}

In this section we gather various results needed for the proof of Theorem 1.7 and for the remarks at the end of this paper.

2.1. Abhyankar's lemma. We will construct unramified covers using Abhyankar's lemma, a version of which we now state. If $\pi: X \rightarrow Y$ and $\phi: Y^{\prime} \rightarrow Y$ are surjective morphisms of curves, then by a compositum of $X$ and $Y^{\prime}$ over $Y$, we mean a curve whose function field is a compositum of $k(X)$ and $k\left(Y^{\prime}\right)$ over $k(Y)$.

Lemma 2.1 (Abyhankar's lemma). Let $\pi: X \rightarrow Y$ and $\phi: Y^{\prime} \rightarrow Y$ be surjective morphisms of curves. Assume that for all closed points $x \in X$ and $y^{\prime} \in Y^{\prime}$ with $\pi(x)=\phi\left(y^{\prime}\right)$, the ramification index of $\phi$ at $y^{\prime}$ divides the ramification index of $\pi$ at $x$ and is not divisible by $p$. Let $X^{\prime}$ be a compositum of $X$ and $Y^{\prime}$ over $Y$. Then $X^{\prime}$ is an unramified cover of $X$.

Proof. This follows from a local version of Abhyankar's lemma, such as [SGA 1, XIII.5.2].

Remark 2.2. Even if $k(X)$ and $k\left(Y^{\prime}\right)$ are linearly disjoint over $k(Y)$, the fiber product $X \times_{Y} Y^{\prime}$ need not be a compositum in our sense, since it could be singular.

\subsection{Modular curves $X_{0}^{*}(\ell)$ of small genus.}

Lemma 2.3. Let $\ell$ be a prime. Let $X_{0}^{*}(\ell)$ be the quotient of the modular curve $X_{0}(\ell)$ over $\overline{\mathbb{Q}}$ (or over any field of characteristic not divisible by $\ell$ ) by its AtkinLehner involution. Let $g$ be the genus of $X_{0}^{*}(\ell)$. Then

$$
\begin{aligned}
& g=0 \Longleftrightarrow \ell \in\{2,3,5,7,13,23,29,31,37,41,47,59,71\} \\
& g=1 \Longleftrightarrow \ell \in\{11,17,19,37,43,53,61,79,83,89,101,131\} \\
& g=2 \Longleftrightarrow \ell \in\{67,73,103,107,167,191\} .
\end{aligned}
$$

If $g>2$, then Aut $X_{0}^{*}(\ell)_{\overline{\mathbb{Q}}}$ is trivial.

Proof. The values of $\ell$ for which $g \leq 2$ can be deduced by combining the list of $X_{0}(\ell)$ for which $g\left(X_{0}(\ell)\right) \leq 1$ (by the general formula, these are the primes $\ell \leq 19)$, the list of hyperelliptic $X_{0}(\ell)$ [Ogg1974], the list of bielliptic $X_{0}(\ell)$ [Bars1999], and the list of hyperelliptic $X_{0}^{*}(\ell)$ [Hasegawa-Hashimoto1996]. The final statement is proved in [Baker-Hasegawa2003]. 
Remark 2.4. In fact, the papers cited above together with [Hasegawa1997] contain the information needed to list all (not necessarily prime) $\ell \in \mathbb{Z}_{>0}$ with $g\left(X_{0}^{*}(\ell)\right)=0,1,2$.

2.3. Existence of covers of $\mathbb{P}^{1}$ unramified outside 3 points. The following lemma is well known. It was used, for instance, in [Darmon-Granville1995] to prove that $x^{p}+y^{q}=z^{r}$ has at most finitely many pairwise relatively prime integer solutions for any fixed $p, q, r \in \mathbb{Z}_{>1}$ with $1 / p+1 / q+1 / r<1$.

Lemma 2.5. Let $k$ be an algebraically closed field of characteristic 0. Let $n_{0}, n_{1}, n_{\infty} \in \mathbb{Z}_{>1}$. Then there exists a Galois cover $X \rightarrow \mathbb{P}_{k}^{1}$ unramified outside $0,1, \infty$ and with ramification indices exactly $n_{0}, n_{1}, n_{\infty}$ above $0,1, \infty$ respectively.

Proof. We elaborate on the suggestion in the paragraph before Proposition 3a in [Darmon-Granville1995] to use results stated in [SerreTopicsInGaloisTheory]. By [SerreTopicsInGaloisTheory, Theorem 6.3.3], it suffices to construct the cover for $k=\mathbb{C}$. Let $\pi_{1}$ be the topological fundamental group of $\mathbb{P}_{\mathbb{C}}^{1}-\{0,1, \infty\}$, and let $s_{0}, s_{1}, s_{\infty}$ be the monodromy generators at the three points. Let $N$ be the smallest normal subgroup of $\pi_{1}$ containing $s_{0}^{n_{0}}, s_{1}^{n_{1}}, s_{\infty}^{n_{\infty}}$. By [SerreTopicsInGaloisTheory, Theorem 6.4.2] (with $s=3, t=0$ ), the images of $s_{0}, s_{1}, s_{\infty}$ in $\pi_{1} / N$ have orders exactly $n_{0}, n_{1}, n_{\infty}$. By the last paragraph of [SerreTopicsInGaloisTheory, Section 6.3], the map from $\pi_{1}$ to its profinite completion is injective, so $\pi_{1}$ contains a normal subgroup $N^{\prime}$ of finite index such that the images of $s_{0}, s_{1}, s_{\infty}$ in $\pi_{1} / N^{\prime}$ have orders exactly $n_{0}, n_{1}, n_{\infty}$. By [SerreTopicsInGaloisTheory, Theorem 6.1.4], the analytic covering of $\mathbb{P}_{C}^{1}-\{0,1, \infty\}$ corresponding to $N^{\prime}$ is an algebraic curve $X_{0}$. The corresponding smooth projective curve $X$ is the desired Galois covering of $\mathbb{P}^{1}$.

\section{Proof of the main theorem}

3.1. Case 1: $g(Y) \in\{1,2\}$. If $g(Y)=2$, then $Y$ is hyperelliptic and $X \Rightarrow Y$, so there is nothing to show. So assume that $Y$ is an elliptic curve $E$. Since $g(X) \geq 2, X$ is ramified above some point of $E$, which we may assume is the identity of $E$. Let $e$ be a prime dividing the ramification index there. Replace $X \rightarrow E$ by its (unramified) base extension by the multiplication-by- $\ell$ map $E \rightarrow$ $E$ for some prime $\ell \geq 5$ not equal to $p$ (and choose an irreducible component if necessary, so that the new $X$ is again a curve). Thus we reduce to the case where $X \rightarrow E$ has ramification index divisible by $e$ above each $\ell$-torsion point of $E$. Fix a Weierstrass model of $E$. The $\ell^{2}-1$ nonzero $\ell$-torsion points come in pairs sharing the same $x$-coordinate: let $a_{1}, \ldots, a_{\left(\ell^{2}-1\right) / 2}$ be all these $x$-coordinates.

By Lemma 2.1, a compositum of $X$ and

$$
H: z^{e}=\frac{\left(x-a_{1}\right)\left(x-a_{2}\right)}{\left(x-a_{3}\right)\left(x-a_{4}\right)} .
$$

over $\mathbb{P}^{1}$ (with coordinate $x$ ) gives an unramified cover of $X$ that dominates $H$. The function $z$ on $H$ is of degree 2, and applying the Hurwitz formula to 
$x: H \rightarrow \mathbb{P}^{1}$ shows that $g(H)=e-1$. Thus if $e \geq 3$, then $H$ is hyperelliptic. If $e=2$, instead use

$$
H: z^{2}=\frac{\left(x-a_{1}\right)\left(x-a_{2}\right)\left(x-a_{3}\right)}{\left(x-a_{4}\right)\left(x-a_{5}\right)\left(x-a_{6}\right)} .
$$

which is hyperelliptic of genus 2 .

We assume $Y \simeq \mathbb{P}^{1}$ from now on.

By the Hurwitz formula, there are $\geq 3$ branch points.

3.2. Case 2: $G$ is solvable. We use induction on \#G. If $H \subsetneq G$ is a nontrivial normal subgroup, then depending on whether $g(X / H) \geq 2, g(X / H)=1$, or $g(X / H)=0$, we apply the inductive hypothesis to $X / H \rightarrow Y$, Case 2 to $X \rightarrow$ $X / H$, or the inductive hypothesis to $X \rightarrow X / H$, respectively. Thus we may assume that $G$ is simple. But $G$ is solvable, so $G \simeq \mathbb{Z} / \ell \mathbb{Z}$ for some prime $\ell \neq p$. Thus $X$ is a $\mathbb{Z} / \ell \mathbb{Z}$-cover of $\mathbb{P}^{1}$. If $\ell=2$ then $X$ itself is hyperelliptic, so assume $\ell \geq 3$.

If we take a compositum with a $\mathbb{Z} / \ell \mathbb{Z}$-cover $\mathbb{P}^{1} \rightarrow \mathbb{P}^{1}$ ramified above exactly two branch points of $X \rightarrow \mathbb{P}^{1}$, we find a new $\mathbb{Z} / \ell \mathbb{Z}$-cover $X^{\prime} \rightarrow \mathbb{P}^{1}$. By Lemma 2.1, $X^{\prime}$ is unramified over $X$. Since $g\left(X^{\prime}\right)>g(X)$, the $\mathbb{Z} / \ell \mathbb{Z}$-cover $X^{\prime} \rightarrow \mathbb{P}^{1}$ is ramified above $\geq 4$ points of $\mathbb{P}^{1}$. Let $x$ be a parameter on $\mathbb{P}^{1}$ whose values $a_{1}, \ldots, a_{4}$ at these points are not $\infty$. Applying Lemma 2.1 to a compositum with the $\mathbb{Z} / \ell \mathbb{Z}$-cover $H \rightarrow \mathbb{P}^{1}$ given by

$$
H: y^{\ell}=\frac{\left(x-a_{1}\right)\left(x-a_{2}\right)}{\left(x-a_{3}\right)\left(x-a_{4}\right)}
$$

shows that $X^{\prime} \Rightarrow H$. And $H$ is hyperelliptic.

3.3. Case 3: There are two branch points whose associated ramification indices have a nontrivial common factor. Let $e$ be a prime dividing the ramification indices above two branch points, and let $e^{\prime}$ be a prime dividing the ramification index above some other branch point $y^{\prime}$. A compositum of $X \rightarrow \mathbb{P}^{1}$ with a $\mathbb{Z} / e \mathbb{Z}$-cover $\phi: \mathbb{P}^{1} \rightarrow \mathbb{P}^{1}$ branched above exactly the first two branch points is a Galois cover $X^{\prime}$ of (a new) $\mathbb{P}^{1}$, and $X^{\prime}$ is unramified over $X$. The new cover $X^{\prime} \rightarrow \mathbb{P}^{1}$ has ramification index $e^{\prime}$ above each of the $e$ points in $\phi^{-1}\left(y^{\prime}\right)$. In particular, $e^{\prime}$ divides the ramification indices above two branch points of the new cover, so we can repeat the process to obtain an infinite commutative (though not necessarily cartesian) diagram

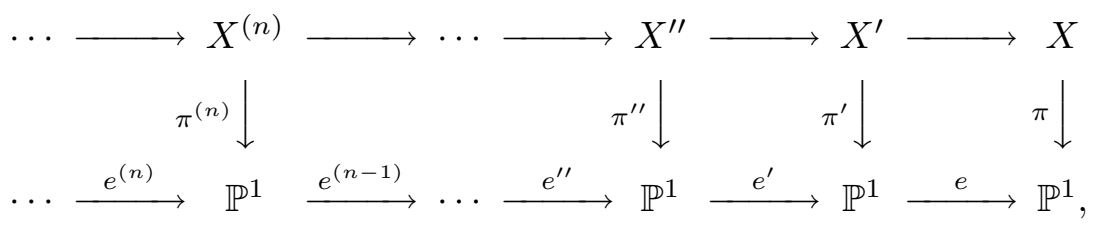

in which the integers $e^{(n)}$ indicate the degrees of cyclic covers. By commutativity, the degree of $X^{(n)} \rightarrow X$ is at least $e^{(n-1)} \cdots e^{\prime} e /(\operatorname{deg} \pi)$, which tends to $\infty$, so $X^{(n+1)} \rightarrow X^{(n)}$ must be of degree $>1$ for infinitely many $n$. Since $g(X) \geq 2$ and 
all morphisms are separable, it follows that $g\left(X^{(n)}\right) \rightarrow \infty$ as $n \rightarrow \infty$. On the other hand, $\operatorname{deg} \pi^{(n)} \leq \operatorname{deg} \pi$, so by the Hurwitz formula, the number of branch points of $\pi^{(n)}$ tends to $\infty$. The ramification indices are bounded by that of $\pi$, so for some $n$, there is an integer $\ell \geq 2$ that is the ramification index above more than 6 branch points. Let $S$ be a $\mathbb{Z} / \ell \mathbb{Z}$-cover of $\mathbb{P}^{1}$ branched above 6 points, with ramification index $\ell$ above each. Applying Lemma 2.1 to a compositum of $X^{(n)}$ and $S$ over $\mathbb{P}^{1}$ shows that $X^{(n)} \Rightarrow S$. Hence $X \Rightarrow S$. The Hurwitz formula shows that $g(S) \geq 2$. Also, by construction, $p \nmid \ell$, so $S$ is tamely superelliptic. By Case $3, S \Rightarrow H$ for some hyperelliptic curve $H$. By transitivity, $X \Rightarrow H$.

3.4. Case 4: Ramification divisible by $2,3, \ell$. By Case 3 , we may assume $\ell \geq 5$. The modular curve $X(\ell)$ is a Galois cover of $\mathbb{P}^{1}$ ramified above three points, with ramification indices $2,3, \ell$. We may assume those three points are the same of the branch points for $X \rightarrow \mathbb{P}^{1}$. Let $Z$ be a compositum of $X$ and $X(\ell)$ over $\mathbb{P}^{1}$. By Lemma 2.1, $Z$ is unramified over $X$. Also $Z$ is Galois over $X(\ell)$.

Suppose $\ell=5$. By the Hurwitz formula, the original cover $X \rightarrow \mathbb{P}^{1}$ must have had either a fourth branch point $P$, or else extra ramification (more than $2,3,5$, respectively) above one of the three branch points $P$. In either case, the preimages of $P$ under $X(5) \rightarrow \mathbb{P}^{1}$ are branch points of $Z \rightarrow X(5)$ having the same ramification index $>1$, so Case 3 shows that $Z \Rightarrow H$ for some hyperelliptic curve $H$. Then $X \Rightarrow Z \Rightarrow H$.

Thus we may assume $\ell \geq 7$. We have $X \Rightarrow X(\ell)$ (through $Z$ ). Since $X(\ell)$ is a solvable cover of the modular curve $X_{0}(\ell)$, we are done by Case 2 if $g\left(X_{0}(\ell)\right) \leq 2$. Otherwise, let $X_{0}^{*}(\ell)$ be the quotient of $X_{0}(\ell)$ by its Atkin-Lehner involution. If $g\left(X_{0}^{*}(\ell)\right) \leq 2$, we apply Case 2 to $X_{0}(\ell) \rightarrow X_{0}^{*}(\ell)$.

Summing up, we are done whenever $g\left(X_{0}^{*}(\ell)\right) \leq 2$. These primes $\ell$ are given by Lemma 2.3. This completes the proof of Theorem 1.7 .

\section{Final remarks}

Remark 4.1. Here we show that in order to prove Theorem 1.7 in characteristic 0 without making any of the additional assumptions (1) through (4), it would suffice to do the case of Galois covers of $\mathbb{P}^{1}$ with non-abelian simple Galois group, ramified above exactly 3 points, above which the ramification indices are distinct primes $p_{1}, p_{2}, p_{3}$.

First exclude cases already covered by Theorem 1.7. Choose three branch points (we may assume they are $0,1, \infty$ on $\mathbb{P}^{1}$ ) and primes $p_{1}, p_{2}, p_{3}$ dividing the associated ramification indices. The $p_{i}$ will be distinct, since otherwise apply Case 3. If $\left\{p_{1}, p_{2}, p_{3}\right\}=\{2,3,5\}$, apply Case 4. Lemma 2.5 gives a Galois cover $Z \rightarrow \mathbb{P}^{1}$ ramified above exactly these three branch points, and with ramification indices $p_{1}, p_{2}, p_{3}$. Since $1 / p_{1}+1 / p_{2}+1 / p_{3}<1$, the Hurwitz formula gives $g(Z)>1$. Applying Lemma 2.1 to a compositum of $X$ and $Z$ shows that $X \Rightarrow Z$, so we have reduced to proving the result for $Z \rightarrow \mathbb{P}^{1}$. Finally, apply induction as in Case 2 to reduce to the case of a simple Galois group (no new primes are introduced into ramification indices during the induction). 
Remark 4.2. In the previous remark, if $Z_{1}$ and $Z_{2}$ are two Galois covers of $\mathbb{P}^{1}$ each ramified above exactly 3 points with ramification indices $p_{1}, p_{2}, p_{3}$, then Lemma 2.1 applied to a compositum of $Z_{1}$ and $Z_{2}$ over $\mathbb{P}^{1}$ shows that $Z_{1} \Leftrightarrow Z_{2}$.

\section{Acknowledgements}

I thank Matt Baker for suggesting the references for the proof of Lemma 2.3.

\section{References}

[Baker-Hasegawa2003] Matthew Baker and Yuji Hasegawa, Automorphisms of $X_{0}^{*}(p)$, J. Number Theory 100 (2003), 72-87. MR1971247 (2004c:11100)

[Bars1999] Francesc Bars, Bielliptic modular curves, J. Number Theory 76 (1999), 154-165. MR1688168 (2000d:11078)

[Belyi1979] G. V. Belyı̆, Galois extensions of a maximal cyclotomic field, Izv. Akad. Nauk SSSR Ser. Mat. 43 (1979), 267-276, 479. MR534593 (80f:12008) (Russian)

[Bogomolov-Husemoller2000] Fedor Bogomolov and Dale Husemöller, Geometric properties of curves defined over number fields, 2000, Preprint MPIM2000-1 at http://www .mpim-bonn.mpg.de/html/preprints/preprints.html.

[Bogomolov-Tschinkel2002-unramified] Fedor Bogomolov and Yuri Tschinkel, Unramified correspondences, Algebraic Number Theory and Algebraic Geometry, Contemp. Math., vol. 300, Amer. Math. Soc., Providence, RI, 2002, pp. 17-25. MR1936365 (2003k:14032)

[Bogomolov-Tschinkel2004-couniformization] __ Couniformization of curves over number fields (2004), 43-57.

[Chevalley-Weil1930] C. Chevalley and A. Weil, Un théorème d'arithmétiques sur les courbes algébriques, Comptes Rendus Hebdomadaires des Séances de l'Acad. des Sci., Paris 195 (1930), 570-572.

[Darmon-Granville1995] Henri Darmon and Andrew Granville, On the equations $z^{m}=F(x, y)$ and $A x^{p}+B y^{q}=C z^{r}$, Bull. London Math. Soc. 27 (1995), 513-543. MR1348707 (96e:11042)

[Hasegawa1997] Yuji Hasegawa, Hyperelliptic modular curves $X_{0}^{*}(N)$, Acta Arith. 81 (1997), 369-385. MR1472817 (99a:11075)

[Hasegawa-Hashimoto1996] Yuji Hasegawa and Ki-ichiro Hashimoto, Hyperelliptic modular curves $X_{0}^{*}(N)$ with square-free levels, Acta Arith. 77 (1996), 179-193. MR1411031 (97m:11082)

[Ogg1974] Andrew P. Ogg, Hyperelliptic modular curves, Bull. Soc. Math. France 102 (1974), 449-462. MR0364259 (51 \#514)

[SerreTopicsInGaloisTheory] Jean-Pierre Serre, Topics in Galois theory, Research Notes in Mathematics, vol. 1, Jones and Bartlett Publishers, Boston, MA, 1992, ISBN 0-86720210-6, Lecture notes prepared by Henri Damon [Henri Darmon]; With a foreword by Darmon and the author. MR1162313 (94d:12006)

[SGA 1] Revêtements étales et groupe fondamental (SGA 1), Documents Mathématiques (Paris) [Mathematical Documents (Paris)], 3, Société Mathématique de France, Paris, 2003, ISBN 2-85629-141-4, Séminaire de géométrie algébrique du Bois Marie 1960-61. [Geometric Algebra Seminar of Bois Marie 1960-61]; Directed by A. Grothendieck. With two papers by M. Raynaud. Updated and annotated reprint of the 1971 original [Lecture Notes in Math., 224, Springer, Berlin]. MR2017446 (2004g:14017) (French) USA

Department of Mathematics, University of California, Berkeley, CA 94720-3840,

E-mail address: poonen@math.berkeley.edu

$U R L:$ http://math. berkeley.edu/ poonen 